\title{
artigo
}

Morais, J. D., Felipe, G. F., Silva, A. A. A., Franco, A. L., Desiderio, G. A., Nobre, M. S., Ucó, M. V., Djedjo, E., Pontes, I. E. A.

Os efeitos da pandemia no comportamento sexual dos indivíduos acometidos por covid-19

\section{Os efeitos da pandemia no comportamento sexual dos indivíduos acometidos por covid-19}

\author{
The effects of the pandemic on the sexual behavior of individuals affected by covid-19 \\ Los efectos de la pandemia en el comportamiento sexual de los individuos afectados por covid-19
}

\section{RESUMO}

Objetivo: Analisar os efeitos da pandemia no comportamento sexual dos indivíduos com COVID-19. Método: Trata-se de uma pesquisa descritiva de corte transversal, quantitativa, composta por 807 pessoas com vida sexual ativa. Resultados: Observou-se, que em sua maioria as pessoas eram casadas e não mudaram o status do relacionamento durante a pandemia com parceiro fixo e que tiveram sua vida sexual afetada pela pandemia $(58,5 \%)$, que continham percepção satisfatória da vida sexual antes da pandemia $(80,5 \%)$, mas que houve uma diminuição durante a pandemia $(23 \%, p=0,001)$. Ocorreu também uma diminuição ao atingir o orgasmo $(60,5 \%)$ para durante a pandemia $(44,5 \%)$. A grande maioria afirmou que a excitação durante a pandemia não mudou $(46,5 \%)$ e apresentaram pouco desconforto/dor durantes as relações sexuais nesse período. Conclusão: A pandemia afetou não somente no comportamento sexual, como também na saúde em geral dos indivíduos.

DESCRITORES: População. Comportamento sexual. Coronavírus.

\section{ABSTRACT}

Objective: To analyze the effects of the pandemic on the sexual behavior of individuals with COVID-19. Method: This is a descriptive cross-sectional, quantitative survey of 807 people with an active sex life. Results: It was observed, that mostly people were married and not change their relationship status during the pandemic with fixed partner and who had their sexual life affected by the pandemic. Results: It was observed, that mostly people had their sex life affected by the pandemic (58.5\%), that they contained satisfactory perception of sex life before the pandemic $(80.5 \%)$, but that there was a decrease during the pandemic $(23 \%, \mathrm{p}=0.001)$. There was also a decrease in reaching orgasm $(60.5 \%)$ to during the pandemic $(44.5 \%)$. The great majority affirmed that the excitement during the pandemic did not change $(46.5 \%)$ and presented little discomfort/ pain during sexual relations in this period. Conclusion: Of pandemic affected not only the sexual behavior, but also the general health of individuals. DESCRIPTORS: Population. Sexual behavior. Coronavírus.

\section{RESUMEN}

Objetivo: Analizar los efectos de la pandemia en el comportamiento sexual de los individuos con COVID-19. Método: Se trata de una investigación descriptiva de corte transversal, cuantitativa, compuesta por 807 personas con vida sexual activa. Resultados: Se observó, que en su mayoría las personas estaban casadas y no cambiaron de estado civil durante la pandemia con pareja estable y que tenían su vida sexual afectada por la pandemia (58,5\%), que tenían una percepción satisfactoria de la vida sexual antes de la pandemia (80,5\%), pero que hubo una disminución durante la pandemia $(23 \%$, $\mathrm{p}=0,001)$. También hubo una disminución en el alcance del orgasmo $(60,5 \%)$ a durante la pandemia $(44,5 \%)$. La gran mayoría afirmó que la excitación durante la pandemia no cambió $(46,5 \%$ ) y presentó poco descontento/dolor durante las relaciones sexuales en este período. Conclusión: La pandemia afectó no sólo al comportamiento sexual, sino también a la salud general de los individuos.

DESCRIPTORES: Población; Comportamiento sexual; Coronavirus

RECEBIDO EM: 08/06/21 APROVADO EM: 14/06/21

\section{Jairo Domingos de Morais}

Fisioterapeuta, Doutor em Modelos de Decisão e Saúde pela UFPB. Professor do Instituto de Ciências da Saúde da UNILAB. ORCID: 0000-0002-8383-7871.

\section{Gilvan Ferreira Felipe}

Enfermeiro, Doutor em Enfermagem pela UECE. Professor do Instituto de Ciências da Saúde da UNILAB.

ORCID: 0000-0003-0674-4396.

\section{Antônia Antonieta Alves da Silva}

Graduanda do curso de Farmácia da UNILAB.

ORCID: 0000-0001-5467-8202.. 


\section{Ana Lydia Franco}

Graduanda do curso de Farmácia da UNILAB.

ORCID: 0000-0001-8632-4177.

\section{Gabriel Alves Desiderio}

Graduando do curso de Farmácia da UNILAB.

ORCID: 0000-0002-0337-5110.

\section{Matheus de Sousa Nobre}

Graduando do curso de Farmácia da UNILAB.

ORCID: 0000-0001-8632-4177.

\section{Miguel Vicente Ucó}

Graduando do curso Enfermagem da UNILAB.

ORCID: 0000-0002-4183-0444.

\section{Elbin Djedjo}

Graduando do curso Enfermagem da UNILAB.

ORCID: 0000-0003-1404-7398.

\section{Isabelle Eunice Albuquerque Pontes}

Fisioterapeuta, Doutora em Saúde Materno Infantil pelo IMIP/PE Professora do Departamento de Fisioterapeuta da Universidade Estadual da Paraíba (UEPB).

ORCID: 0000-0002-2194-8971.

\section{INTRODUÇÃO}

A tualmente, o mundo ainda não conseguiu dimensionar com precisão o impacto que a pandemia do novo Coronavírus atingiu a população em todas as áreas da vida humana mediante sua capacidade de transmissão e letalidade, o que desconfigurou e trouxe mudanças em todas as camadas e estruturas populacionais 1 .

Observa-se uma intensa catástrofe no que concerne à mudança brusca das relações humanas, corroborando ainda mais para uma condição deletéria das relações humanas. Logo, ao analisar esse contexto pelo prisma das relações sexuais, percebe-se uma mudança catastrófica nos diferentes níveis que envolvem as relações sexuais, sejam elas em nível casual ou não2.

Deste modo, observa-se que ao longo dos meses decorrentes do isolamento social, por um lado uma diminuição das relações sexuais aliada também a uma diminuição do prazer sexual e em consonância, um aumento substancial das questões do trato psicológico3. É perceptível que as relações sexuais foram em alguns casos in
Observa-se que ao longo dos meses

decorrentes do

isolamento social,

por um lado uma

diminuição das

relações sexuais

aliada também a

uma diminuição do

prazer sexual e em

consonância, um

aumento substancial

das questões do

trato psicológico tensificadas ou diminuídas, dependendo do contexto em que esses indivíduos estavam inseridos 4 .

Nessa perspectiva, Yuksel e Ozgor5 apontam um cenário voltado para diminuição do desejo e satisfação sexual entre homens e mulheres além de uma diminuição constante das relações de risco ocasionadas pelo distanciamento social e outro cenário que aponta parte dos entrevistados inclinados a terem um número de parceiros maiores em um contexto pós-pandemia.

Aliado a isso, também é perceptível o aumento substancial da masturbação e visitas a sites pornográficos 6 e na diminuição do desejo sexual no parceiro à medida que se aumenta a percepção do auto-erotismo nas relações individuais7. Outro cenário está justamente no aumento do desejo sexual e no uso do mesmo para a intensificação das relações conjugais e no uso do sexo para o alívio dos transtornos mentais causados pelo distanciamento social8.

Mediante a esse cenário antagônico, é perceptível que as relações sexuais exercem um papel crucial na vida humana9. Contudo, ainda não é possível dimensionar com precisão o impacto do distanciamento so- 


\section{artigo}

Morais, J. D., Felipe, G. F., Silva, A. A. A., Franco, A. L., Desiderio, G. A., Nobre, M. S., Ucó, M. V., Djedjo, E., Pontes, I. E. A.

Os efeitos da pandemia no comportamento sexual dos indivíduos acometidos por covid-19

cial nas relações sexuais, entretanto, é necessário a elaboração de estudos em diferentes contextos afim de traçar um panorama mais geral do impacto do distanciamento na vida sexual de homens e mulheres. Logo, para tal entendimento, o presente estudo tem como objetivo em analisar os efeitos da pandemia na saúde e no comportamento sexual dos indivíduos com confirmação de COVID-19.

\section{MÉTODOS}

Trata-se de um estudo descritivo de corte transversal e abordagem quantitativa que faz parte da Pesquisa multicêntrica, de âmbito Nacional realizada no mês de junho de 2020 por meio de um questionário online pelo Google Forms e divulgada através das mídias sociais. Foi realizado um recorte da amostra, por acometimento de $\mathrm{CO}$ VID-19, e que foram agrupadas em indivíduos com COVID-19 $(\mathrm{n}=99)$ e aqueles sem COVID-19 ( $\mathrm{n}=708)$, compondo um total de 807 indivíduos maiores de 18 anos com vida sexual ativa das 5 regióes brasileiras.

Utilizou o pacote estatístico IBM - SPSS 22.0 para análise dos dados e para buscar associação entre as variáveis realizou a regressão logística binária através da razão de chances (Odds Ratio ajustado) a fim de investigar associação entre a orientação sexual e as variáveis independentes do estudo com intervalo de confiança de $95 \%$ e com nível de significância de $5 \%$ (p-valor $<0,05)$.

$\mathrm{O}$ estudo seguiu as recomendações dos preceitos éticos da Resolução $\mathrm{n}^{\circ}$ 466/2012, do Conselho Nacional de Saúde sendo aprovado pelo Comitê de Ética e Pesquisa (CEP) da Universidade da Integração Internacional da Lusofonia Afro-Brasileira (UNILAB) sob o CAAE $\mathrm{n}^{\circ} 31383120.7 .0000 .5576$ e parecer $\mathrm{n}^{\mathrm{o}}$ $4.050 .129 / 2020$.

\section{RESULTADOS}

A prevalência de pessoas que tiveram sintomas ou confirmação de COVID-19 na amostra estudada foi de $12 \%$, tendo em sua maioria mulheres com idade entre
18 e 40 anos, pardas, pós-graduandas que trabalham e possuem renda familiar entre 3 e 5 salários-mínimos (Tabela 1). Houve associação significativa estatisticamente entre apresentar sintomas ou confirmação de COVID-19 e as variáveis escolaridade e renda familiar

Observou-se, no que diz respeito aos aspectos relacionais e de orientação sexual dos indivíduos, que apresentaram ou não sinais e sintomas de COVID-19, em sua maioria Heterossexuais, casados ou em

Tabela 1 - Perfil sociodemográfico dos indivíduos com ou sem COVID-19.

\begin{tabular}{|c|c|c|c|c|c|c|c|}
\hline \multicolumn{2}{|c|}{ Variáveis } & \multicolumn{2}{|c|}{ COM } & \multicolumn{2}{|c|}{ SEM } & \multirow{2}{*}{$\begin{array}{l}\text { Total } \\
\%\end{array}$} & \multirow{2}{*}{$\begin{array}{l}\mathrm{P}- \\
\text { valor }\end{array}$} \\
\hline \multirow{4}{*}{ Sexo } & & $\mathrm{N}$ & $\%$ & $\mathrm{~N}$ & $\%$ & & \\
\hline & Masculino & 24 & 3,0 & 177 & 21,9 & 24,9 & 0,969 \\
\hline & Feminino & 68 & 8,4 & 485 & 60,1 & 68,5 & \\
\hline & Não Informado & 7 & 0,9 & 46 & 5,7 & 6,6 & \\
\hline \multirow[t]{2}{*}{ Idade } & $\begin{array}{l}\text { Entre } 18 \text { e } 40 \\
\text { anos }\end{array}$ & 85 & 10,5 & 601 & 74,5 & 85,0 & 0,881 \\
\hline & $>40$ anos & 14 & 1,7 & 107 & 13,3 & 15,0 & \\
\hline \multirow{6}{*}{ Etnia } & Negra & 8 & 1,0 & 58 & 7,2 & 8,2 & 0,108 \\
\hline & Parda & 47 & 5,8 & 266 & 33,0 & 38,8 & \\
\hline & Branca & 41 & 5,1 & 370 & 45,8 & 50,9 & \\
\hline & Indígena & 0 & 00,0 & 7 & 00,9 & 0,9 & \\
\hline & Oriental Asiático & 1 & 0,1 & 4 & 00,5 & 0,6 & \\
\hline & Outro & 2 & 0,2 & 3 & 00,4 & 0,6 & \\
\hline \multirow{7}{*}{ Escolaridade } & $\begin{array}{l}\text { Ensino } \\
\text { Fundamental } \\
\text { Completo }\end{array}$ & 0 & 00,0 & 1 & 0,1 & 0,1 & $0,005^{*}$ \\
\hline & $\begin{array}{l}\text { Ensino Médio } \\
\text { incompleto }\end{array}$ & 2 & 0,2 & 0 & 00,0 & 00,2 & \\
\hline & $\begin{array}{l}\text { Ensino Médio } \\
\text { completo }\end{array}$ & 1 & 0,1 & 21 & 2,6 & 2,7 & \\
\hline & Ensino Técnico & 0 & 00,00 & 11 & 1,4 & 01,4 & \\
\hline & $\begin{array}{l}\text { Ensino Superior } \\
\text { incompleto }\end{array}$ & 17 & 2,1 & 147 & 18,2 & 020,3 & \\
\hline & $\begin{array}{l}\text { Ensino Superior } \\
\text { completo }\end{array}$ & 22 & 2,7 & 166 & 20,6 & 023,3 & \\
\hline & Pós-graduação & 57 & 7,1 & 362 & 44,9 & 051,9 & \\
\hline \multirow{3}{*}{ Trabalha } & Sim & 81 & 10,1 & 533 & 66,0 & 76,1 & 0,153 \\
\hline & Não & 18 & 2,2 & 175 & 21,7 & 23,9 & \\
\hline & $\begin{array}{l}\text { < } 1 \text { salário } \\
\text { mínimo }\end{array}$ & 3 & 0,4 & 18 & 2,2 & 2,6 & $0,017^{*}$ \\
\hline \multirow{3}{*}{ Renda } & $\begin{array}{l}1 \text { a } 3 \text { salários } \\
\text { mínimos }\end{array}$ & 26 & 3,2 & 224 & 27,8 & 31,0 & \\
\hline & $\begin{array}{l}3 \text { a } 5 \text { salários } \\
\text { mínimos }\end{array}$ & 41 & 5,1 & 187 & 23,2 & 28,3 & \\
\hline & $\begin{array}{l}>5 \text { salários } \\
\text { mínimos }\end{array}$ & 29 & 3,6 & 279 & 34,6 & 38,2 & \\
\hline
\end{tabular}


união estável que possuem entre 3 e 5 anos de relação com apenas um parceiro fixo e que tal relação não mudou durante a pandemia. Não houve associação estatística entre as variáveis relacionais e de orientação sexual com ter ou não sintomas/confirmação de COVID-19 (Tabela 2).

Os indivíduos que tiveram sintomas/ confirmação de COVID-19 apresentaram em sua maioria sua vida sexual afetada pela pandemia $(58,5 \%)$, ressalta-se que tais sujeitos apresentaram percepção satisfatória da vida sexual antes da pandemia $(80,5 \%)$ mas que ocorreu uma diminuição dessa avaliação durante a pandemia em $23 \%$ $(\mathrm{p}=0,001)$. Ocorreu também uma redução da frequência com que conseguiam atingir o orgasmo durante as relações sexuais antes da pandemia $(60,5 \%)$ para durante a pandemia $(44,5 \%)$. O estudo encontrou ainda que os indivíduos que tiveram $\mathrm{CO}$ VID-19 possuem 2 vezes mais chances de ter mudado a percepção satisfatória da sua vida sexual antes da pandemia para durante a pandemia (Tabela 3).

A grande maioria afirmou que a excitação sexual durante esse período não mudou (46,5\%) durante a pandemia, apresentando uma frequência de poucas vezes de dor ou desconforto durante ou após as relações sexuais $(73,5 \%)$ e já o grau de desejo durante a pandemia foi considerado moderado a alto $(30 \%-31 \%$ respectivamente). Houve associação significativa estatisticamente entre apresentar sintomas ou confirmação de COVID-19 e a frequência de desconforto ou dor durante ou após as relações sexuais no período de pandemia $(\mathrm{p}=0,046)$.

\section{DISCUSSÃO}

O presente estudo buscou compreender como a pandemia da COVID-19 afetou a vida sexual dos indivíduos cujo perfil dos indivíduos com sintomas e/ou confirmação de COVID-19 demonstrou uma distribuição proporcional, entre homens e mulheres, uniforme o que corrobora com os achados encontrados pela Organização Mundial de Saúde (OMS) ao apresentar a distribuição mundial entre os casos confirmados da doença10.
Tabela 2 - Aspectos relacionais dos indivíduos com ou sem COVID-19.

\begin{tabular}{|c|c|c|c|c|c|c|}
\hline \multirow[t]{2}{*}{ Variáveis } & & \multicolumn{2}{|c|}{ Sim } & \multicolumn{2}{|c|}{ Não } & \multirow[t]{2}{*}{ p-valor } \\
\hline & & $\mathrm{N}$ & $\%$ & $\mathbf{N}$ & $\%$ & \\
\hline \multirow{4}{*}{ Orientação Sexual } & $\begin{array}{l}\text { Heterosse- } \\
\text { xual }\end{array}$ & 81 & 10,0 & 576 & 71,4 & \multirow{4}{*}{0,928} \\
\hline & $\begin{array}{l}\text { Homosse- } \\
\text { xual }\end{array}$ & 10 & 1,2 & 77 & 9,5 & \\
\hline & Assexual & 0 & 0 & 3 & 0,4 & \\
\hline & Bissexual & 8 & 1,0 & 50 & 6,2 & \\
\hline \multirow{5}{*}{$\begin{array}{l}\text { Tipo de } \\
\text { relacionamento }\end{array}$} & Outros & 0 & 0 & 2 & 0,2 & \multirow{5}{*}{0,852} \\
\hline & Solteiro & 31 & 3,8 & 197 & 24,4 & \\
\hline & Namorando & 25 & 3,1 & 200 & 24,8 & \\
\hline & $\begin{array}{l}\text { Casado } \\
\text { ou união } \\
\text { estável }\end{array}$ & 43 & 5,3 & 306 & 37,9 & \\
\hline & Viúvo & 0 & 0 & 3 & 0,4 & \\
\hline \multirow{3}{*}{$\begin{array}{l}\text { Status do } \\
\text { relacionamento mudou } \\
\text { na pandemia }\end{array}$} & Outros & 0 & 0 & 2 & 0,2 & \multirow{3}{*}{0,208} \\
\hline & Sim & 10 & 1,2 & 89 & 11,0 & \\
\hline & Não & 47 & 5,8 & 661 & 81,9 & \\
\hline \multirow{2}{*}{$\begin{array}{l}\text { Tempo com o parceiro } \\
\text { fixo }\end{array}$} & Sim & 77 & 9,5 & 531 & 65,8 & \multirow{2}{*}{0,548} \\
\hline & Não & 22 & 2,7 & 177 & 21,9 & \\
\hline \multirow{5}{*}{$\begin{array}{l}\text { Tempo com o parceiro } \\
\text { fixo }\end{array}$} & $<6$ meses & 18 & 2,2 & 80 & 9,9 & \multirow{5}{*}{0,237} \\
\hline & $\begin{array}{l}6 \text { meses e } 1 \\
\text { ano }\end{array}$ & 21 & 2,6 & 133 & 16,5 & \\
\hline & $\begin{array}{l}\text { Entre } 1 \text { e } 3 \\
\text { anos }\end{array}$ & 14 & 1,7 & 93 & 11,5 & \\
\hline & $\begin{array}{l}\text { Entre } 3 \text { e } 5 \\
\text { anos }\end{array}$ & 25 & 3,1 & 231 & 28,6 & \\
\hline & $>5$ anos & 21 & 2,6 & 171 & 21,1 & \\
\hline
\end{tabular}

Tabela 3 - Vida sexual dos indivíduos com ou sem sintomas/confirmação de COVID-19.

\begin{tabular}{|c|c|c|c|c|c|}
\hline \multicolumn{2}{|l|}{ Variāveis } & Sim & Não & OR (IC 95\%) & p-valor \\
\hline & & $\mathbf{N}$ & N & & \\
\hline \multirow{2}{*}{ Vida sexual afetada } & Sim & 58 & 385 & \multirow{2}{*}{$\begin{array}{c}1,132 \\
(0,665-1,926)\end{array}$} & \multirow{2}{*}{0,648} \\
\hline & Não & 41 & 323 & & \\
\hline \multirow{2}{*}{$\begin{array}{l}\text { Percepção da vida sexual } \\
\text { antes da pandemia }\end{array}$} & Satisfatória & 80 & 558 & \multirow{2}{*}{$\begin{array}{c}0,797 \\
(0,565-1,123)\end{array}$} & \multirow{2}{*}{0,194} \\
\hline & Insatisfatória & 19 & 150 & & \\
\hline \multirow{2}{*}{$\begin{array}{l}\text { Houve mudança na } \\
\text { avaliação da percepção } \\
\text { da vida sexual durante a } \\
\text { pandemia }\end{array}$} & Sim & 57 & 286 & \multirow[t]{2}{*}{$\begin{array}{c}2,002 \\
(1,308-3,066)\end{array}$} & \multirow[t]{2}{*}{$0,001^{*}$} \\
\hline & Não & 42 & 422 & & \\
\hline
\end{tabular}




\section{artigo}

Morais, J. D., Felipe, G. F., Silva, A. A. A., Franco, A. L., Desiderio, G. A., Nobre, M. S., Ucó, M. V., Djedjo, E., Pontes, I. E. A.

Os efeitos da pandemia no comportamento sexual dos indivíduos acometidos por covid-19

No que diz respeito a faixa etária observou que o grupo de 18-40 anos apresentou a grande maioria dos casos confirmados e que apresenta consonância com os resultados apontado por Gouveia et al. (2020)11 que, na cidade do Ceará, apresentou predominância da faixa etária entre 20 e 59 anos. As pessoas acometidas por COVID-19 no presente estudo são em maioria pardas seguidas das brancas o que corrobora com Ferreira e colaboradores 12 ao encontrar mesmo público acometido.

O presentou estudo encontrou no tocante a escolaridade, indivíduos com ensino superior e pós-graduação cujo fator este que demonstra de importante relevância uma vez que a escolaridade pode estar ligada a classe social e, portanto, sugerindo hábitos e condições de vida que podem representar um fator de risco ou proteção para disseminação e conhecimento sobre as doenças virais infecciosas 13. Os indivíduos do presente estudo, em sua maioria, trabalham e apresentam renda entre 3 e 5 salários mínimos o que se observa como fator preponderante no prognóstico da doença e diz respeito aos impactos importantes que refletem diretamente no acesso a moradia, apoio social, tratamento e recursos para adquirir sua proteção e combate ao vírus.

Os indivíduos possuem uma certa estabilidade no que diz respeito aos aspectos relacionais como o tipo de relacionamento, parceiro fixo e não ter mudado de relacionamento durante a pandemia, o que pode apresentar como fatores de apoio social diante das implicações negativas e das consequências do distanciamento social ocasionado pela pandemia. $\mathrm{O}$ apoio social é visto como um fator de proteção cuja o apoio familiar é significantemente associado a comportamentos de promoção da saúde e bem-estar 14 .

No que tange a prevalência de sintomas ou confirmação de COVID-19, a pandemia contribuiu para a diminuição do nível de satisfação da vida sexual e da frequência de orgasmos encontrando anteriormente para durante a pandemia. Resultados semelhantes foram encontrados por Karagöz e colaboradores 15 que analisaram a sexualidade de casais na Turquia e que constatou

\begin{tabular}{|c|c|c|c|c|c|}
\hline \multirow{3}{*}{$\begin{array}{l}\text { Atingia orgasmo antes da } \\
\text { pandemia }\end{array}$} & $\begin{array}{l}\text { Sim, todas às } \\
\text { vezes }\end{array}$ & 31 & 274 & 1 & \multirow{3}{*}{0,312} \\
\hline & $\begin{array}{l}\text { Sim, boa } \\
\text { parte das } \\
\text { vezes }\end{array}$ & 60 & 392 & $\begin{array}{c}0,739 \\
(0,467-1,171)\end{array}$ & \\
\hline & Não & 8 & 42 & $\begin{array}{c}0,594 \\
(0,256-1,379)\end{array}$ & \\
\hline \multirow{3}{*}{$\begin{array}{l}\text { Atingiu orgasmo durante a } \\
\text { pandemia }\end{array}$} & $\begin{array}{l}\text { Sim, todas às } \\
\text { vezes }\end{array}$ & 25 & 239 & 1 & \multirow{3}{*}{0,219} \\
\hline & $\begin{array}{l}\text { Sim, boa } \\
\text { parte das } \\
\text { vezes }\end{array}$ & 44 & 267 & $\begin{array}{c}0,635 \\
(0,377-1,069)\end{array}$ & \\
\hline & Não & 30 & 202 & $\begin{array}{c}0,704 \\
(0,401-1,237)\end{array}$ & \\
\hline \multirow{3}{*}{$\begin{array}{l}\text { Sente/ Sentia Excitação } \\
\text { sexual durante a pandemia }\end{array}$} & $\begin{array}{l}\text { Sim, não } \\
\text { mudou }\end{array}$ & 46 & 400 & 1 & \multirow{3}{*}{0,138} \\
\hline & $\begin{array}{l}\text { Sim, aumen- } \\
\text { tou }\end{array}$ & 33 & 205 & $\begin{array}{c}0,714 \\
(0,443-1,152)\end{array}$ & \\
\hline & Não & 20 & 103 & $\begin{array}{c}0,592 \\
(0,336-1,045)\end{array}$ & \\
\hline \multirow{5}{*}{$\begin{array}{l}\text { Grau de desejo ou interesse } \\
\text { sexual durante a pandemia }\end{array}$} & $\begin{array}{l}\text { Muito baixo } \\
\text { ou nenhum }\end{array}$ & 9 & 27 & 1 & \multirow{5}{*}{0,134} \\
\hline & Baixo & 11 & 82 & $\begin{array}{c}1,285 \\
(0,686-2,408)\end{array}$ & \\
\hline & Moderado & 31 & 266 & $\begin{array}{c}1,457 \\
(0,782-2,717)\end{array}$ & \\
\hline & Alto & 30 & 227 & $\begin{array}{c}1,266 \\
(0,576-2,827)\end{array}$ & \\
\hline & Muito Alto & 18 & 106 & $\begin{array}{c}0,509 \\
(0,206-1,259)\end{array}$ & \\
\hline \multirow{4}{*}{$\begin{array}{l}\text { Frequência de desconforto } \\
\text { ou dor durante ou após as } \\
\text { relações sexuais no período } \\
\text { de pandemia }\end{array}$} & $\begin{array}{l}\text { Não Houve } \\
\text { relação }\end{array}$ & 15 & 151 & 1 & \multirow{4}{*}{$0,046^{*}$} \\
\hline & $\begin{array}{l}\text { Sempre } \\
\text { ou quase } \\
\text { sempre }\end{array}$ & 2 & 13 & $\begin{array}{c}0,646 \\
(0,133-3,136)\end{array}$ & \\
\hline & Muitas vezes & 9 & 25 & $\begin{array}{c}0,276 \\
(0,109-0,698)\end{array}$ & \\
\hline & Poucas vezes & 73 & 519 & $\begin{array}{c}0,706 \\
(0,394-1,267)\end{array}$ & \\
\hline
\end{tabular}

a diminuição da satisfação em comparação com o período pré-pandêmico e bem como a frequência de orgasmos. Somado a isso, constatou-se no presente estudo que indivíduos que contraíram a COVID-19 tiveram até 2 vezes mais chance de terem a sua percepção da satisfação afetada pela doença em sua vida sexual durante a pandemia.
Além disso, encontrou-se que a excitação sexual não mudou durante a pandemia e o grau de desejo variou de moderado a alto, o mesmo encontrado na pesquisa com os casais Turcos. Esse comportamento pode ser explicado pela hipótese de que contrair a doença e o isolamento social fez com que os indivíduos permanecessem mais tempo em 
casa o que resultou que o desejo e algumas variáveis sexuais aumentaram visivelmente. Entretanto, houve uma frequência maior de dores e desconfortos durante ou após as relações sexuais nesse período, tendo um resultado significativo quando associado a presença de sintomas ou a confirmação da doença.

\section{CONCLUSÕES}

Com base nos achados do presente estudo, foi possível concluir que a pandemia de COVID-19 afetou a vida sexual dos indivíduos que tiveram sintomas ou confirmação da doença, trazendo uma diminuição no nível de satisfação sexual e no orgasmo dos mesmos. Pode-se inferir também, que o grau de excitação sexual dessas pessoas se manteve constante antes e depois da pandemia, no entanto, a maioria desse grupo apresentou algum tipo de dor ou desconforto durante a relação.

\section{REFERÊNCIAS}

1. Zurlo MC, Cattaneo Della Volta MF, Vallone F. COVID-19 Student Stress Questionnaire: Development and Validation of a Questionnaire to Evaluate Students' Stressors Related to the Coronavirus Pandemic Lockdown. Front Psychol. 2020 Oct 22; 11:576758.

2. Pascoal PM, Raposo CF, Roberto MS. A Transdiagnostic Approach to Sexual Distress and Sexual Pleasure: A Preliminary Mediation Study with Repetitive Negative Thinking. Int J Environ Res Public Health. 2020 Oct 27;17(21):7864.

3. Li W, Li G, Xin C, Wang Y, Yang S. Challenges in the practice of sexual medicine in the time of COVID-19 in China. J Sex Med. 2020; 17 (7): $1225-1228$.

4 - Pascoal PM, Raposo CF, Pelixo P, Pinto P. COVID-19 e sexualidade recomendações para a intervenção psicológica. SPSC. 2021 jan: 1-11.

5. Yuksel B, Ozgor F. Effect of the COVID-19 pandemic on female sexual behavior. Int J Gynaecol Obstet. 2020 Jul;150(1):98-102.

6. Ballester-Arnal, Rafael \& Nebot-Garcia, Juan Enrique \& Ruiz, Estefanía \& Giménez-García, Cristina \& Gil Llario, María. (2020). "INSIDE" Project on Sexual Health in Spain: Sexual Life During the Lockdown Caused by COVID-19. Sexuality Research and Social Policy Journal of NSRC.

7. Cocci A, Giunti D, Tonioni C, Cacciamani G, Tellini R, Polloni G, Cito G, Presicce F, Di Mauro M, Minervini A, Cimino S, Russo Gl. Love at the time of the Covid-19 pandemic: preliminary results of an online survey conducted during the quarantine in Italy. Int J Impot Res. 2020 Sep;32(5):556-557.

8. Carvalho J, Pereira R, Barreto D, Nobre PJ. The Effects of Positive Versus Negative Mood States on Attentional Processes During Exposure to Erotica. Arch Sex Behav. 2017 Nov;46(8):2495-2504.
9. Sousa, T.J.; Oliveira, D.F.; Estrela, F.M.; Soares da Silva, A.V.; David, R.A.R.; Rosa, D.O.S.; Neves da Silva, G.; Lassala, J.N.; Fernandes, A.P.; Sexualidade e autoestima dos pacientes com úlceras diabéticas. Saúdecoletiva. 2021; 11(67): 6775-6781.

10. World Health Organization. Gender and covid19: Advocacy brief. 2020.

11. Gouveia PMG, Gouveia SV, Sousa VS, Silva MSE, Medeiros RG, Lago da Silva R. Incidência e letalidade da covid-19 no ceará, 2020. Cadernos ESP [Internet]. $22^{\circ}$ de julho de 2020 [citado $27^{\circ}$ de junho de 2021];14(1):10-16. Disponivel em: //cadernos.esp.ce.gov.br/index.php/cadernos/article/view/326

12. Ferreira ADS, Perovano LS, Barboza LI, Nascimento WM, Silva FM, Reis ECD. Perfil sociodemográfico dos pacientes confirmados para covid-19 residentes no espírito santo, brasil. AtoZ: Novas Práticas em Informação e Conhecimento, 2020 dez 03; 9(2): 216-223.

13. Lenzi L, Wiens A, Grochocki MHC, Pontarolo R.. Study of the relationship between socio-demographic characteristics and new influenza A (H1N1). Brazilian Journal of Infectious Diseases [online]. 2011 oct; [Accessed 26 Junho 2021]; 15(5): 457-461. Disponivel em:https://www.scielo.br/j/bjid/a/VNXy7xrG5bRfVqyMKtw7DVy/ abstract/?lang=en

14. Chew BH, Khoo EM, Chia YC. Social support and glycemic control in adult patients with type 2 diabetes mellitus. Asia Pac J Public Ealth. 2015;27(2):NP166-73.

15 - Karagöz MA, Gul A, Borg C, Erihan $\odot$ B, Uslu M, Ezer M et al. Influência da pandemia de COVID-19 na sexualidade: um estudo transversal entre casais na Turquia . Jornal internacional de pesquisa sobre impotência. 10 de novembro de 2020. 